\title{
Simulation for Fuzzy PID Temperature Control System
}

\author{
Zhengqiang Guan ${ }^{1, a}$, Xiaoming Luo ${ }^{2, b}$, Lepeng Song ${ }^{1, c}$ \\ ${ }^{1}$ Chongqing University of science and technology, Chongqing, China \\ asjkgzq@126.com, ${ }^{\mathrm{c}} \mathrm{s}$ Iphq@163.com \\ ${ }^{2}$ Property management co., Itd. Shengzhen Merchants., Shengzhen city, Guangdong, China \\ b70luo@163.com
}

Keywords: Fuzzy PID; proportional integral differential parameter adjustment; temperature control; heating system

\begin{abstract}
For the current general heating control system tracks the set temperature hysteresis heating device to automatically adjust the real-time problem of poor design a fuzzy self-tuning proportional-integral-derivative (PID) controller parameters. Scoop out using PID control and fuzzy control algorithm combined method; fuzzy PID control parameters are adjusted. Use Matlab to model in simulink, the aryl the controller simulation analysis. The results show that the fuzzy self-tuning PID controller overshoot $\sigma \approx 1 \%$, steady-state error Island $\mathrm{e}_{\mathrm{s}}=0$. This method can improve the performance of the temperature control system.
\end{abstract}

\section{Introduction}

Fried heating process such as the study of tea machine, tea machine fried heating system must be adapted to process fried tea, temperature control accuracy and efficiency directly affects the quality of tea , such a heating system is very time-varying system, the required temperature small hysteresis control system to keep track of the set target temperature.

Proportional Integral Derivative (Proportion Integration Differentiation, PID) control is still the most widely used control law, it is because of the simple PID controller structure to meet the requirements of industrial process control, PID control especially strong so that it can be more robust changes good fit a wide range of process conditions. However, PID may be because they belong essentially linear control [1-4], and large overshoot of the heating system such a big lag, nonlinear, time-varying systems, parameter tuning to optimize the value of the value of just having some localized, so the overall effect is not controlled ideal. PID control can get better control effect, required by adjusting the proportional, integral and derivative three kinds of control, the formation of both the mutual cooperation between the amount of control and mutual checks and balances. When pure fuzzy control deviation and the deviation in the rate of change as input, the control process is not smooth phenomenon sometimes occurs, there are steady-state error. This paper presents fuzzy self-tuning PID controller parameters, the fuzzy control PID controller parameters for heating temperature control system tuning.

\section{Fuzzy control theory}

Fuzzy controller is based on the knowledge of the controller, which uses fuzzy rules to control the expression of experiences and constitute the rule base, through the use of fuzzy logic or fuzzy inference rule base control experience and knowledge, so as to achieve the purpose of control. Specifically includes three components: fuzzy processing, reconciliation fuzzy reasoning. 
Fuzzy processing is to determine the value of the fuzzy controller input conversion process variable values [5-7] in response to vague language, the language of these response variable values [8] by the corresponding membership function $\mathrm{OK}$.

Uncertainty reasoning methods for fuzzy system called fuzzy inference methods. Fuzzy reasoning is often used an approximate reasoning method, also known as the "likelihood inference "is a fuzzy set membership function transformation and calculation process. It is a prerequisite for making vague, fuzzy control rules generated by the theory. Baldwin reasoning is used here. Since fuzzy sets can not be precise analog or digital control systems, and therefore must be precise calculation. The fuzzy set in these most significant representatives of the determined value [9], as the system output control. In this paper, the center of gravity method is the weighted average method.

\section{Fuzzy self-tuning PID controller design}

Fuzzy self-tuning PID Controller Structure. Fuzzy self-tuning PID controller is the fuzzy control rules for PID parameters to modify an adaptive control system. To error and error rate of change of $\mathrm{E}$ $\mathrm{EC}$ as input to meet the different moments of E and EC on PID parameter self-tuning requirements. Using fuzzy control rules on PID parameters changed, they constitute the adaptive fuzzy PID controller, the structure shown in Fig. 1.

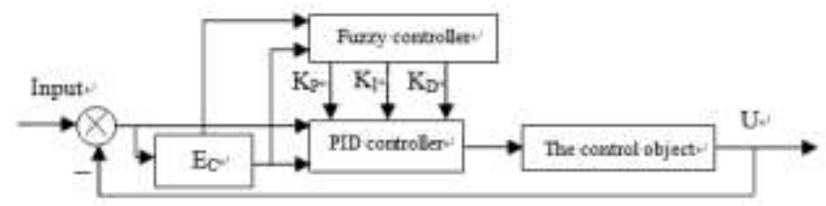

Fig. 1. adaptive fuzzy controller structure

PID Impact on System Performance. From the system's stability, speed of response, overshoot and steady state accuracy and other considerations, $K_{p} K_{i}$ and $K_{d}$ role is as follows :

(1) Scale factor $K_{p}$ role is to accelerate the response speed of the system and improve the regulation accuracy of the system. $\mathrm{K}_{\mathrm{p}}$ bigger, faster response speed of the system, adjust the accuracy of the system is higher, but easy to overshoot, and even lead to system instability. $\mathrm{K}_{\mathrm{p}}$ value is too small, it will reduce the adjustment precision, the response is slow, thus extending regulation time, the system is static and dynamic characteristics deteriorate.

(2) Integral coefficient $K_{i}$ is role is to eliminate the steady-state error. Static error $K_{i}$ bigger, the faster elimination system, but $\mathrm{K}_{\mathrm{i}}$ is too large, the initial response process will produce windup phenomenon, causing a large overshoot response process. If $\mathrm{K}_{\mathrm{i}}$ is too small, $\mathrm{K}_{\mathrm{i}}$ will make the system difficult to eliminate static error, adjustment precision affected system.

(3) The role of the differential control is an issue worthy of discussion. Differential coefficient $\mathrm{K}_{\mathrm{d}}$ is role is to improve the dynamic characteristics of the system, its main function is to suppress the deviation in any direction change in the response process, the deviation change in advance forecast. But Kd advance over the General Assembly to make the process of braking response, thereby extending regulation time, and will reduce the anti-jamming performance of the system. 
Input and Output Membership Functions. Fuzzy parameters regulator is a dual input and three output fuzzy controller, input fuzzy variable most heated oven temperature and the temperature difference between the rate of change of the deviation E EC; output fuzzy variable scaling parameter $K_{p}$, integral parameter $K_{i}$, differential parameter $K_{d}$. Input variables $E$ and Ec fuzzy subset of the language by the seven large negative value of the variable composition of $\{\mathrm{NB}$, negative in $\mathrm{NM}$, negative small NS, zero ZO, are small PS, middle PM, CP PB $\},\{-3$ on the domain , 3$\} .3$ output fuzzy subset \{negative large NB, negative in NM, negative small NS, zero $\mathrm{ZO}$, are small PS, middle $\mathrm{PM}, \mathrm{CP} \mathrm{PB}\}, 3$ outputs universe fuzzy variables are $\{\mathrm{O}, 6)$.

Considering the extent of coverage of the universe, sensitivity and robustness, the membership function of each fuzzy subset are used triangular membership function, with high accuracy, in the form of simple, high efficiency is calculated, taking into account the membership function affect the size of the width of the system, the error is small when compared with the steep shape using the membership function, it has a higher resolution, the error is relatively large when compared with gentle shapes using the membership function, allowing the system to obtain better robustness. The following were given $\mathrm{E}, \mathrm{EC}$ and three output variables $\mathrm{K}_{\mathrm{p}}, \mathrm{K}_{\mathrm{i}}, \mathrm{K}_{\mathrm{d}}$ membership function distribution.

Fuzzy Control Rules. Fuzzy control rules elbow fuzzy control system plays a key role. Design principles of fuzzy control rule is : When the error is large, the control should be such that the error is reduced halo ; When the error is small, you should try to eliminate errors while preventing larger overshoot phenomenon. Specifically, it is time to find out PID3 between different parameters and E and EC fuzzy relationship, that run continuously detect E and EC, according to the fuzzy control principle of three -line parameters modified to meet different E EC control parameters and different requirements, leaving the controlled object has a good dynamic and static performance . Control rules of fuzzy controller is based on the manual control strategy is generally controlled by the operator observing the object from the stability, response speed, overshoot, steady precision and PID system to the system shadow

Ring and other considerations, to establish appropriate fuzzy rule table based on actual experience, because of space limitations, here $\triangle K_{p}$ fuzzy control rule, as shown in Table 1

Fuzzy Reasoning and Fuzzy Decision. Fuzzy reasoning is actually an approximate reasoning, fuzzy reasoning based on fuzzy conditional statement. In the fuzzy control, fuzzy reasoning is the premise of fuzzy decision, but also the theoretical basis of the formation of fuzzy control rules.

\section{Simulation results and analysis}

In order to confirm the reasonableness of the control algorithm, using Matlab, Simulink to build adaptive fuzzy PID controller and a standard PID controller model, and simulation. By using two kinds of different control algorithms, respectively, their respective simulation results obtained, the structure of the system shown in Fig. 2.

Table 1. Fuzzy control table of $K_{P}$ 


\begin{tabular}{llllllll}
\hline $\mathrm{K}_{\mathrm{p}}$ & & & & $\mathrm{ec}$ & & \\
\hline & $\mathrm{NB}$ & $\mathrm{NM}$ & $\mathrm{NS}$ & ZO & PS & PM & PB \\
\hline NB & PB & PB & PM & PM & PS & ZO & ZO \\
NM & PB & PB & PS & PS & ZO & ZO & NS \\
NS & PM & PM & PM & PS & ZO & NS & NS \\
e ZO & PM & PM & PS & ZO & NS & NM & NM \\
PS & PS & PS & ZO & NS & NS & NM & NM \\
PM & PS & ZO & S & NM & NM & NM & NB \\
PB & ZO & ZO & NM & NM & NM & NB & NB \\
\hline
\end{tabular}

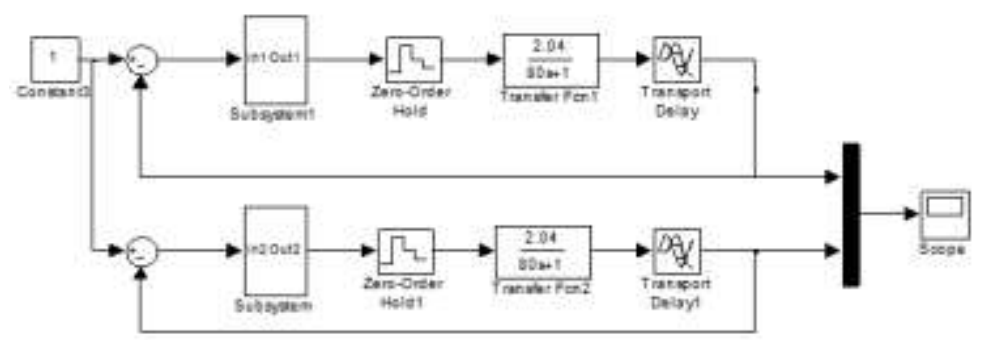

Fig. 2. Fuzzy PID system structure and standard.

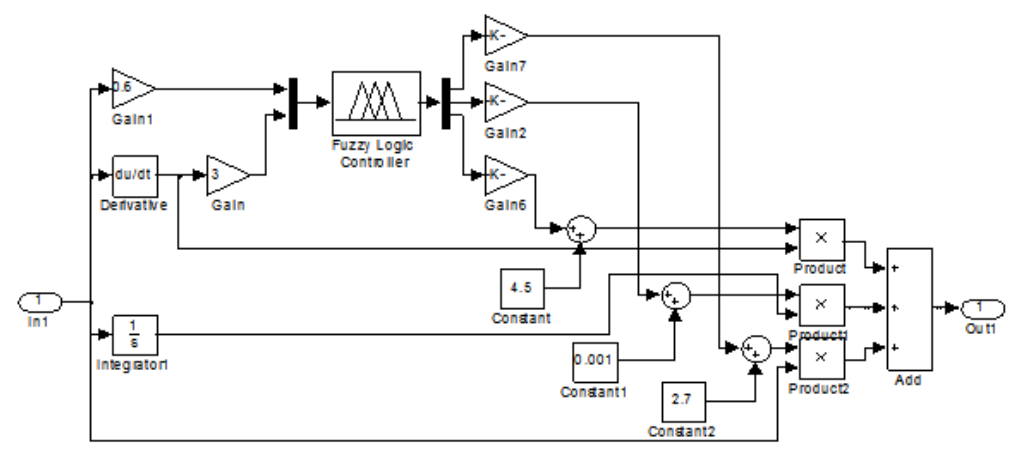

Fig. 3. Fuzzy PID system structure

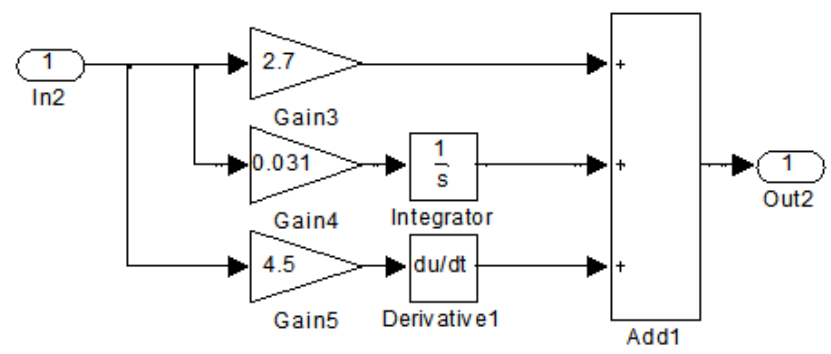

Fig. 4. Standard PID system structure

Figure 3 shows Fuzzy PID system structure. Figure 4 shows Standard PID system structure. Figure 5 shows system step response. In this paper, the system transfer function of the controlled object is $2.04 \mathrm{e}-9 \mathrm{~s} / 80 \mathrm{~s}+1$, respectively, to take fuzzy self-tuning PID algorithm and PID algorithm simulation. Parameters are set as follows: $\mathrm{K}_{\mathrm{p}} 0=2.7, \mathrm{~K}_{\mathrm{i}} 0=0.031, \mathrm{~K}_{\mathrm{d}} 0=4.5$; fuzzy self-tuning PID error and error change rate of the algorithm were taken $\mathrm{Ke}=0.6, \mathrm{Kec}=0.3$, scale factor, respectively $\mathrm{hp}=1.2, \mathrm{hi}=0.3$, hd $=1.7$; system step response simulation results shown in Figure 5, PID control overshoot stare $\sigma=5 \%$, the fuzzy PID overshoot stare $\sigma=1 \%$; settling time ts 2 kinds of control methods $=100 \mathrm{~s}$, the steady-state phase error Island es $=0$ 


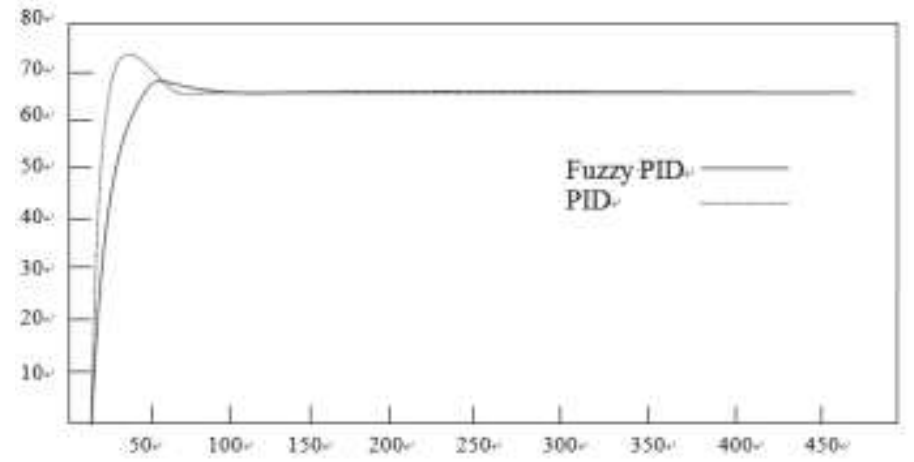

Fig. 5. System step response curve

\section{Conclusion}

When using conventional PID control system has a large overshoot, while pure fuzzy control itself can not eliminate the steady state error. This article will be two kinds of control methods organic junction station, using fuzzy self-tuning PID algorithm overcomes the disadvantages of the PID control, to achieve the ideal performance of the system to adjust short time, small overshoot and steady-state error small

\section{Project Funding}

This research is funded by Research Foundation of The Natural Foundation of Chongqing City, the project No. is cstc2011jjA80022.

This research is funded by Board of Education of The Natural Foundation of Chongqing City, the project No. is KJ131416 and KJ131416.

This research is funded by Research Foundation of Chongqing University of Science \& Technology, the project No. is CK2011Z16.

Corresponding Author: Lepeng Song, Chongqing University of science and technology, School of Electrical and Information Engineering, 401331, Chongqing, China.

\section{Reference}

[1] Lu sweet rainbow. Chen nine fuzzy PID controller and Its Application in Temperature Control System. Chinese Electrical Engineering. 1995, 15 (1): 16.18.

[2] Chang of Ho Shearer fuzzy adaptive control theory and its application. Beijing: University of Aeronautics Off Press, 2001.

[3] Caomao Jun . Pan Li Chi , Xiao word neural network PID parameter adjustment contained in the application. Calculate Machine engineering, 2011, 37 (12) :182-184.

[4] Zhang Puguang microcontroller-based temperature controller did not count and research [D] two security: Xi'an Electronic Science and Technology Learn, 2008:45-51. 
[5] Hangzhou Press advanced PID control and MATLAB. Beijing: Electronic Industry Press .2003:67-80.

[6] Zhang. Jiang ridicule, the array of its workers. PID parameter self-tuning fuzzy controller design and study their electrical and mechanical engineering, 2006, 23 (9):19-21.

[7] Wang Zhulin, in Di Feng, Xu Shenglin air flow servo system Pm fuzzy self -tuning controller. Electrical and Mechanical Engineering, 2009,26 (1) :57 -59.

[8] Ma possession applied research. Yinchuan in the oven SCM temperature control system of fuzzy PID control technology: Second Northwest University for Nationalities, 2007.

[9] Wei Wei Matlab Control Toolbox Technical Manual. Beijing: Guofang industrial publishing male . 2004 